From Astrophysical Implications of the Laboratory Study of Presolar Materials

AIP Conference Proceedings, 1997

T.J. Bernatowicz and E. Zinner (eds.)

\title{
The Propagation and Survival of Interstellar Grains
}

\author{
A.P. Jones ${ }^{1,2}$, A.G.G.M. Tielens ${ }^{3}$, D.J. Hollenbach ${ }^{3}$ \\ and C.F. McKee ${ }^{4}$ \\ ${ }^{1}$ SETI Institute, 2035 Landings Drive, Mountain View, CA 94043, USA \\ ${ }^{2}$ IAS, Université Paris XI - Bâtiment 121, 91405 Orsay cedex, France \\ ${ }^{3}$ NASA Ames Research Center, MS 245-3, Moffett Field, CA 94035, USA \\ ${ }^{4}$ Space Sciences Laboratory, University of California, Berkeley, CA 94720, USA
}

\begin{abstract}
In this paper we discuss the propagation of dust through the interstellar medium (ISM), and describe the destructive effects of stellar winds, jets, and supernova shock waves on interstellar dust. We review the probability that grains formed in stellar outflows or supernovae survive processing in and propagation through the ISM, and incorporate themselves relatively unprocessed into meteoritic bodies in the solar system. We show that very large (radii $\geq 5 \mu \mathrm{m}$ ) and very small grains (radii $\leq 100 \AA$ ) with sizes similar to the pre-solar $\mathrm{SiC}$ and diamond grains extracted from meteorites, can survive the passage through $100 \mathrm{~km} \mathrm{~s}^{-1}$ shock waves relatively unscathed. High velocity $\left(\geq 250 \mathrm{~km} \mathrm{~s}^{-1}\right)$ shocks destroy dust efficiently. However, a small $(\sim 10 \%)$ fraction of the stardust never encountered such fast shocks before incorporation into the solar system. All grains should therefore retain traces of their passage through interstellar shocks during their propagation through the ISM. The grain surfaces should show evidence of processing due to sputtering and pitting due to small grain cratering collisions on the micron-sized grains. This conclusion seems to be in conflict with the evidence from the large grains recovered from meteorites which seem to show little interstellar processing.
\end{abstract}

\section{Introduction}

Dust formed in stellar environments (stardust and circumstellar dust) is primarily made in the shells around stars in the red giant phase of their evolution (e.g., M giants, carbon stars and radio luminous $\mathrm{OH} / \mathrm{IR}$ stars), but some small fraction is 


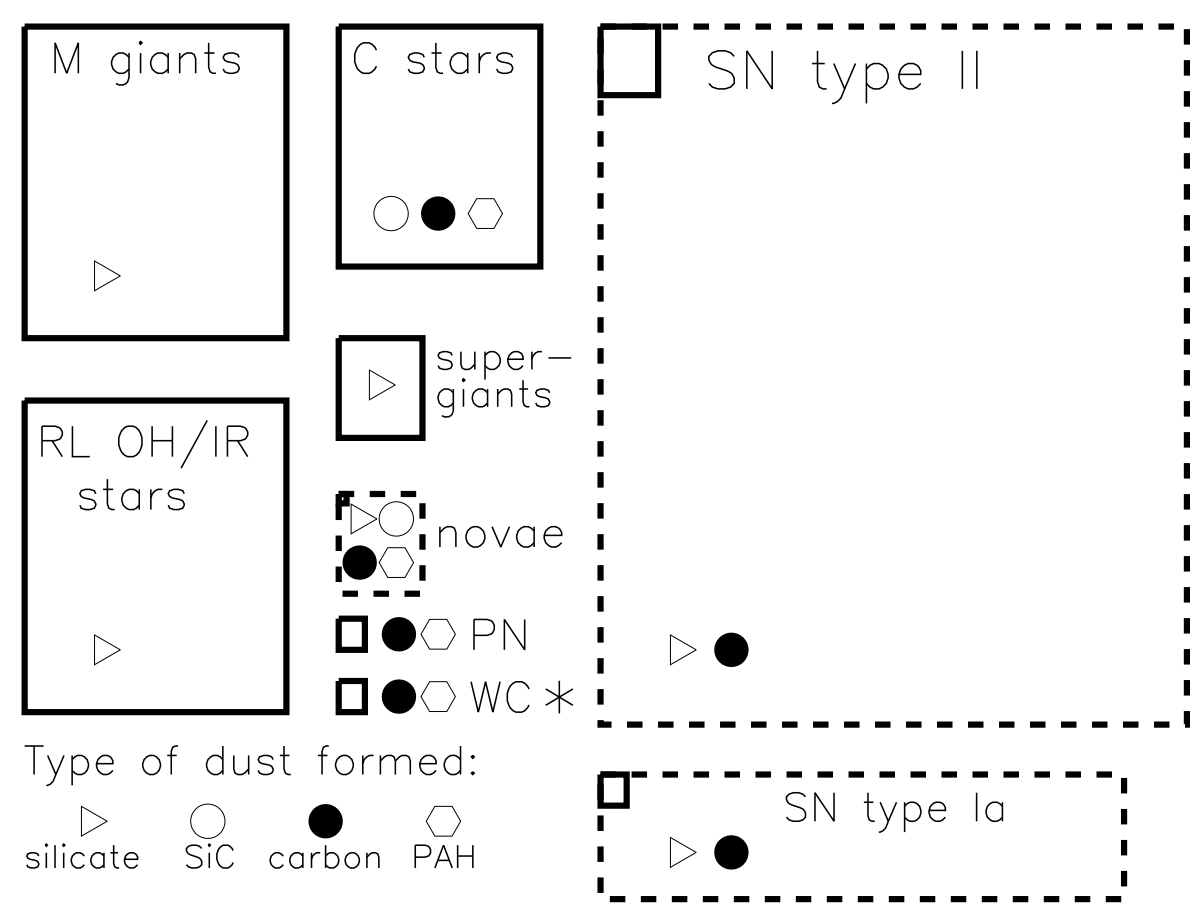

Figure 1: Relative contributions, by boxed area, of stardust sources in the ISM. C stars refers to carbon star sources, and PAH refers to polycyclic aromatic hydrocarbon species. The dashed lines indicate possible upper limit contributions.

also formed in the circumstellar shells around supergiants, novae, planetary nebulae $(\mathrm{PN}), \mathrm{WC}$ stars, and in the ejecta of supernovae (SN types Ia and II). The presence of circumstellar dust is revealed by the large near IR continua arising from the heated dust in the vicinity of the star, and also by the opacity of the circumstellar shell which is due to the absorption and scattering of starlight by the local dust. In Figure 1 we show the relative contributions of the major sources of stardust in the ISM, and in Table 1 we give the stardust source contributions in units of $10^{-6} \mathrm{M}_{\odot}$ $\mathrm{kpc}^{-2} \mathrm{yr}^{-1}[1,2]$.

Stardust is injected into the ambient ISM by stellar winds. Observationally, stars with the highest mass loss rates show the presence of dust, implying a link between high mass loss rates and the formation of dust. In essence, high mass loss rates correspond to high densities near the photosphere which are conducive to dust formation. Radiation pressure pushes the dust out, dragging the gas along, and this coupling is also favored by the high density [3].

In the ISM, dust is subject to processing in a variety of environments. This includes the growth of ice mantles, consisting of simple molecules such as $\mathrm{H}_{2} \mathrm{O}$, $\mathrm{CO}, \mathrm{CO}_{2}$, and $\mathrm{CH}_{3} \mathrm{OH}$, in the shielded environment of molecular clouds, and their subsequent processing into more complex organic mantles when exposed to FUV 
Table 1: Contributions of Stardust Sources in the ISM

\begin{tabular}{lc}
\hline Source & $\begin{array}{c}\text { Contribution } \\
\left(\times 10^{-6} \mathrm{M}_{\odot} \mathrm{kpc}^{-2} \mathrm{yr}^{-1}\right)\end{array}$ \\
\hline M giants & 3 \\
RL OH/IR stars & 3 \\
C stars & 2 \\
Supergiants & 0.2 \\
Novae & $0.003-0.2$ \\
PN & 0.03 \\
WC stars & 0.03 \\
SN type II & $0.15-14$ \\
SN type Ia & $0.03-2.3$ \\
\hline
\end{tabular}

photons, and cosmic ray bombardment. Because of the low temperatures and densities, the dust formed in the ISM - we shall call it "ISM-dust" as opposed to stardust - is formed far from equilibrium and will probably resemble kerogen, in terms of structure, presence of impurities, etc., rather than graphite. Also, because the $\mathrm{Si} / \mathrm{C}$ abundance ratio is much less than unity, no silicates or $\mathrm{SiC}$ grains are expected to form in the ISM (although silicon may well be incorporated into mantle materials [4]). Interstellar dust, the dust which resides in the ISM, is then a mixture of ISM-dust and stardust.

Information on interstellar dust can be gleaned from observations of stellar reddening, spectral absorption features, and the gas phase abundances of (rockforming) elements [5]. In particular, the interstellar grain size distribution is commonly represented by a steep power law $\left(n(a) \propto a^{-3.5}[6], \mathrm{MRN}\right)$ which extends into the molecular domain (i. e., PAHs [7]). Because of the limited amount of dustforming elements, and the fact that such a power law distribution has most of the mass in the largest particles, there must be a maximum grain size cut off beyond which the interstellar grain size distribution steepens considerably [8]. This maximum size is of order $3000 \AA$. There have been many reviews in recent years on the subject of interstellar dust [9-16] and the reader is referred to these, and the references therein, for further details. In this paper, we are mainly concerned with the destruction of dust in the diffuse ISM.

We are most interested in the dust in the warm intercloud component of the three-phase model of the ISM [17], because it is in this phase of the ISM that the bulk of the grain destruction occurs [18-22]. The three-phase model of the ISM consists of a hot intercloud medium, a warm ionized/neutral medium (or "warm intercloud medium"), and the cold neutral medium. For the hot, warm, and cold phases, respectively, the volume filling factors are $\sim 0.6, \sim 0.4$, and $\sim 0.02-0.04$, the mean hydrogen atom densities, $n_{\mathrm{H}}$, are $\sim 0.003, \sim 0.25$, and $\sim 40 \mathrm{~cm}^{-3}$, and the mean gas kinetic temperatures, $T_{\mathrm{k}}$, are $\sim 5 \times 10^{5}, \sim 10^{4}$, and $\sim 80 \mathrm{~K}[17]$. The cold medium rapidly cycles with the warm medium, on time scales of order 
$3 \times 10^{7} \mathrm{yr}$, due to the photodissociation and photoionization of diffuse and molecular clouds by massive stars [21]. The warm medium cycles back to the cold medium by recombination in shielded regions and by shock compression. In a steady state, the warm medium, with about $10 \%$ of the mass, must cycle to the cold medium on time scales of order $3 \times 10^{6} \mathrm{yr}$. The mass in the hot phase is too low for significant grain destruction to occur within the lifetime of a supernova remnant, and the small filling factor and low shock velocities of the cold component ensure little contribution to the grain destruction from this phase. It is therefore grain processing in the warm ISM, through the effects of supernova shock waves, that determines the lifetime of grains in the ISM.

\section{Stardust and ISM-Dust in the Solar System}

In the ISM we have direct evidence for amorphous silicate grain materials (both stardust and ISM-dust) from the $\mathrm{Si}-\mathrm{O}$ bond stretching and bending modes at $10 \mu \mathrm{m}$ and $20 \mu \mathrm{m}$ observed in absorption and emission in dusty stellar envelopes, and in absorption toward stars without dusty circumstellar envelopes. There is evidence for hydrocarbon grains from the $3.4 \mu \mathrm{m} \mathrm{C}-\mathrm{H}$ stretching vibration which is seen in absorption along lines of sight through the diffuse ISM. The bump in the extinction curve at $2175 \AA$ is also attributed to C-bearing dust, generally of graphitic composition. We also have indirect evidence for carbon-bearing dust from the depletion of carbon in the diffuse ISM. It has been suggested [23] that the grains known as GEMS (Glass with Embedded Metal and Sulphides) found in the collected interplanetary dust particles represent silicate stardust transported through the ISM. This suggestion, whilst intriguing, is still not without problems. A detailed overview of the evidence for, and against, GEMS being interstellar material has been presented $[24]$.

Stardust is clearly detected in the solar system. The evidence from meteorites shows that presolar grains were transported through the ISM and incorporated into the solar nebula 4.5 billion years ago. Unequivocally, dust that formed in circumstellar environments and survived for some considerable time in the ISM has been recovered from meteorites [25] (also, see the extensive reviews elsewhere in these proceedings). In Table 2 we summarize the extracted and analyzed refractory presolar grain components of the Orgueil meteorite (data taken from [26] and references therein). The presolar grains described in Table 2 are, however, only trace components making up less than $0.15 \%$ of the meteorite mass. Presumably much of the less refractory ISM-dust and stardust (e.g., silicates) incorporated into the meteorite is lost during the chemical processing used to extract the refractory grains from the bulk meteorite. Interstellar dust that entered into the solar nebula would have been reprocessed through many sublimation and recondensation events in the ISM, and would therefore have lost much of the chemical memory of its circumstellar birthsites.

In addition, to the meteoritic data there is now very strong evidence that the 
Table 2: Pre-solar Grains in the Orgueil Meteorite

\begin{tabular}{lcc}
\hline Composition & Radius & Abundance $(\%$ by mass $)$ \\
\hline Diamond & $\sim 5 \AA$ & 0.145 \\
$\mathrm{SiC}$ & $150 \AA-5 \mu \mathrm{m}$ & 0.0014 \\
Graphite & $0.4-3.5 \mu \mathrm{m}$ & 0.0006 \\
$\mathrm{Al}_{2} \mathrm{O}_{3}$ & $\geq 0.5 \mu \mathrm{m}$ & $5 \times 10^{-5}$ \\
$\mathrm{TiC}$ & $35-100 \AA$ & $\sim 10^{-9}$ \\
\hline
\end{tabular}

solar system is still collecting interstellar grains. The dust detectors on the Ulysses spacecraft which flew by Jupiter in 1992 [27,28] detected sub-micron-sized particles, and the more recent radar studies of particles entering the Earth's atmosphere [29] detected small meteoroids. In both cases the particles had velocities in excess of the escape velocity for the solar system at the point of detection. The detected grains must therefore be of interstellar origin. The Ulysses particles have a measured mass flux of $5 \times 10^{-21} \mathrm{~g} \mathrm{~cm}^{-2} \mathrm{~s}^{-1}$, roughly consistent with the estimated flux of interstellar dust in the solar vicinity [27]. The interstellar particles measured by the Ulysses fly-by of Jupiter have an average radius of $0.4 \mu \mathrm{m}$, slightly larger than typical interstellar grains. The particles entering the Earth's atmosphere that were detected in the radar studies are significantly larger (sizes $\sim 15-40 \mu \mathrm{m}$ ) than those detected by the Ulysses experiments. However, grains smaller than those measured by Ulysses - or typical interstellar grains, for that matter - will be expelled from the inner solar system through the effects of radiation pressure and interaction with the solar wind carried magnetic field [28].

\section{Propagation Processes}

Grains formed in circumstellar shells propagate away from the star through the relatively gentle outward force of radiation pressure. In this process the grains couple with the gas through gas-grain collisions and drive mass loss. The radiation pressure force is counteracted by gas-drag. The net effect of these two forces determines the terminal velocity of the grains, and also the rate of grain growth in the densest parts of the circumstellar shell $[30,31]$. The outward radiation pressure force on the grains is given by,

$$
F_{\mathrm{pr}}=\pi a^{2}\left\langle Q_{\mathrm{pr}}\right\rangle\left(\frac{L_{\star}}{4 \pi r^{2} c}\right),
$$

where $a$ is the grain radius, $\left\langle Q_{\mathrm{pr}}\right\rangle$ is the radiation pressure efficiency factor, $L_{\star}$ is the stellar luminosity, $r$ is the distance from the star, and $c$ is the velocity of light. The radiation pressure efficiency factor is determined by the absorption and scattering efficiency factors, $Q_{a b s}$ and $Q_{s c a}$, respectively, and a scattering asymmetry factor $g$, through the following expression,

$$
\left\langle Q_{\mathrm{pr}}\right\rangle=Q_{\mathrm{abs}}+(1-g) Q_{\mathrm{sca}} .
$$


The parameter $g$ is a measure of the forward or backward-scattering properties of the grains and therefore, in part, determines the effectiveness of the coupling between the stellar radiation scattered by the grains and their outward motion. The gas drag force is approximately given by

$$
F_{\mathrm{d}}=\pi a^{2} \rho_{\text {gas }} v_{\mathrm{d}}^{2}
$$

where $\rho_{\text {gas }}$ is the gas density, and $v_{\mathrm{d}}$ is the dust drift velocity with respect to the gas. The stellar mass loss rate is given by

$$
\dot{M}=4 \pi \rho_{\text {gas }} r^{2} v
$$

where $v$ is terminal velocity of the gas, and we can then write the dust drift velocity (cf. [32]) as ,

$$
v_{\mathrm{d}} \simeq\left(\frac{\left\langle Q_{\mathrm{pr}}\right\rangle L_{\star} v}{\dot{M} c}\right)^{1 / 2} \simeq 1.9\left(\frac{a}{500 \AA}\right)^{1 / 2} \mathrm{~km} \mathrm{~s}^{-1}
$$

In Equation (5) we have adopted typical cool stellar radiation field parameters $\left(L_{\star}\right.$ $=5000 \mathrm{~L}_{\odot}, \dot{M}=10^{-5} \mathrm{M}_{\odot} \mathrm{yr}^{-1}$, and $v=10 \mathrm{~km} \mathrm{~s}^{-1}$ ), and a radiation pressure efficiency appropriate for silicates $\left(\left\langle Q_{\mathrm{pr}}\right\rangle \simeq 0.02 a / 500 \AA\right)$. Carbonaceous particles have slightly higher efficiencies $\left(\left\langle Q_{\mathrm{pr}}\right\rangle \simeq 0.08 a / 500 \AA\right)$ and hence slightly higher drift velocities. From Equation (5) we see that the drift velocity increases with decreasing mass loss rate (i. e., density at the condensation radius) and for a mass loss rate of $10^{-8} \mathrm{M}_{\odot} \mathrm{yr}^{-1}$ reaches a limiting value of $\simeq 40 \mathrm{~km} \mathrm{~s}^{-1}$ for $500 \AA$ silicate grains. At high drift velocities there is reduced dust-gas coupling and for lower mass loss rates there is not enough momentum flux in the dust to drive the gas in the form of a wind [32]. In terms of the gas and dust balance of the ISM, the relatively few sources with the highest mass loss rates $\left(>10^{-5} \mathrm{M}_{\odot} \mathrm{yr}^{-1}\right)$ can dominate dust input to the ISM [33]. Typical drift velocities for these types of envelopes are of order $1 \mathrm{~km} \mathrm{~s}^{-1}$, which is less than the threshold velocity for the sputtering of interstellar dust materials (typically $30 \mathrm{~km} \mathrm{~s}^{-1}[34]$ ). Sputtering destruction of the grains by impacting gas atoms in these environments is therefore unimportant. However, the lower threshold velocities $\left(\simeq 2 \mathrm{~km} \mathrm{~s}^{-1}\right)$ for shattering in grain-grain collisions can lead to the fragmentation of graphitic or silicate grains, but will be unimportant for the more resistant $\mathrm{SiC}$ or diamond grains [35].

Further processing can occur in the shock front $\left(v_{\mathrm{s}} \simeq 5-20 \mathrm{~km} \mathrm{~s}^{-1}\right)$ where the ejecta merge with the ISM. Neglecting shattering, the processing of dust in the transition region between the stellar wind and the ISM has been considered [36], and it has been concluded that there is little dust destruction in these environments. Our studies [35] show that, while shattering has a larger effect, the overall destruction and disruption is only minor in such low velocity shocks. Thus, it seems that stellar winds generally provide a gentle mechanism for dust propagation into the ISM.

Supernova shock waves are very efficient at propagating grains through the ISM because they sweep up interstellar gas and dust, and accelerate the material to velocities of order tens to hundreds of kilometers per second. These shocks provide 
the kinetic energy that maintain the turbulent motions in the ISM, and are thus responsible for the turbulent diffusion of dust in the ISM. Most of the mass of dust is in cold clouds, both atomic and molecular; the shocks in these clouds are typically of order $10 \mathrm{~km} \mathrm{~s}^{-1}$, fast enough to shatter about $1-10 \%$ of the largest grains but not to destroy them. The shocks in the warm, intercloud medium are faster, and the large differential gas-grain and grain-grain velocities that are generated behind the shock front can lead to grain destruction and reprocessing. Thus, shock waves are essential in causing the dust to diffuse through the ISM, but not all dust survives this process. The destructive and disruptive effects of shock waves on dust are considered in detail in the next section. In general the effects of stellar jets on grains will be similar to the shocks considered below. However, the densities of the interaction regions will be higher than shocks in the warm medium, and the mass of the ISM affected by jets will be smaller than that affected by supernovae.

\section{Dust Survival in Shock Waves}

In shock waves, energetic grain-grain collisions and collisions between gas atoms/ions and dust grains lead to the loss of grain mass to the gas, and to changes in the grain size distribution. In Figure 2, we show the shock structure and postshock grain velocities for a $100 \mathrm{~km} \mathrm{~s}^{-1}$ shock traversing the warm intercloud phase of the ISM $[22,37]$. The charged grains in the postshock gas undergo betatron acceleration as they gyrate around the magnetic field lines. As the postshock gas cools and compresses, the magnetic field increases, which in turn leads to the increased gyration speeds of the grains through the gas. In Figure 2, two stages of betatron acceleration can be seen, each associated with increasing gas density. Betatron acceleration is opposed by collisional and plasma drag forces which try to bring the grains to rest with respect to the gas. At the higher grain velocities in Figure 2 the dominant drag force is that due to collisions with the gas, and varies as $(a \rho)^{-1}$, where $\rho$ is the grain material density. Thus, small and low-density grains are least affected by betatron acceleration and will be most resistant to destruction in shocks.

High energy collisions between gas atoms/ions and grains result in sputtering of the grain surfaces, i. e., the removal of grain surface species by sufficiently energetic collisions of atoms and ions. The sputtering may be thermal, due the high random kinetic velocities of the atoms and ions in the hot postshock gas, or inertial, due to the relative velocity of the betatron-accelerated grains with respect to the gas. The latter sputtering process is often referred to as non-thermal sputtering, but in this work we will use the more descriptive term of inertial sputtering.

Differential grain-grain velocities arise from the $(a \rho)^{-1}$ size and grain density dependence of the collisional drag of the gas atoms/ions on the grains. Also, the grains are gyrating around the magnetic field lines and even without these drag effects the grains would have differential velocities. In general, for the regions of a shock where grain destruction occurs, the velocity differential between grains increases with the difference in their radii (see Figure 2). Grain-grain collisions can 


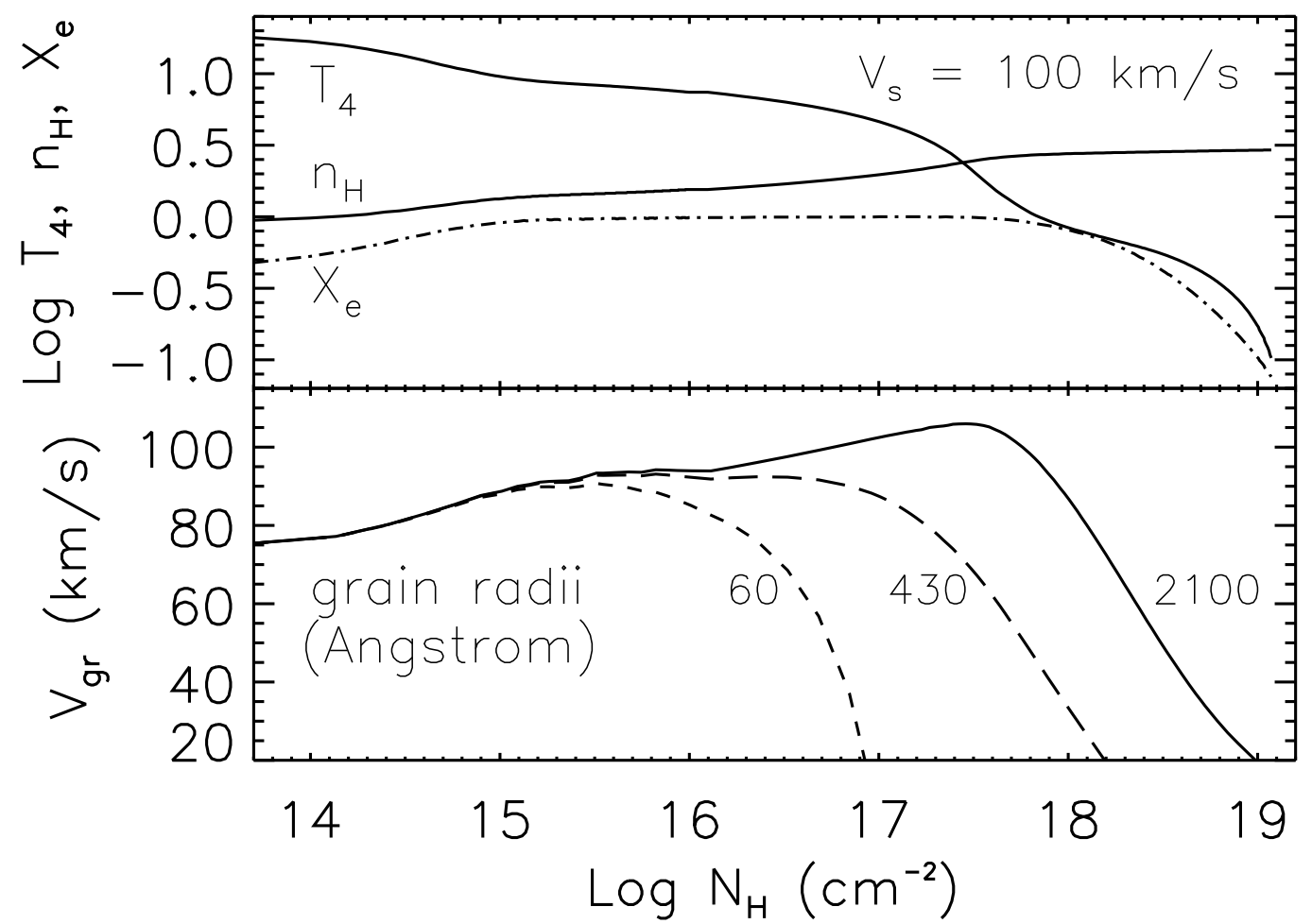

Figure 2: $100 \mathrm{~km} \mathrm{~s}^{-1}$ shock profile (temperature, $T_{4}=T_{\mathrm{k}} / 10^{4} \mathrm{~K}$, density, $n_{\mathrm{H}}\left(\mathrm{cm}^{-3}\right)$, and electron relative abundance, $\left.X_{\mathrm{e}}\right)$ as a function of the shocked column density $N_{\mathrm{H}}$. Also shown in the lower plot are the graphite grains velocities as a function of the shocked column density for three grain radii.

lead both to vaporization, the transfer of grain mass to the gas as atoms/ions, and to shattering, the break-up of the colliding grains into smaller but distinct sub-grains (fragments). The velocity thresholds for vaporization and shattering are typically of order $20 \mathrm{~km} \mathrm{~s}^{-1}$ and $2 \mathrm{~km} \mathrm{~s}^{-1}$, respectively [34,35]. The effects of shattering dominate over those of vaporization, and can lead to major redistributions of the grain mass $[35,38]$.

In keeping with previous work [35], we use the term "destruction" to refer to the sputtering and vaporization processes that result in the transfer of grain mass to the gas; we use the term "disruption" for the shattering process, in which the total grain mass is preserved but redistributed over the grain size distribution. Disruption is any process where a grain is broken down into smaller fragments, and thus includes the fragmentation of "solid" particles and the disaggregation or breakup of porous, aggregated or cluster particles.

There have been many theoretical studies of the effects of supernova shock waves on interstellar dust $[18,19,39-43]$. We have undertaken a theoretical study of the effects of steady state, radiative, J shocks on the dust in the warm ISM $[22,34,35,37]$. As discussed above, it is in the warm medium where interstellar grain destruction by 


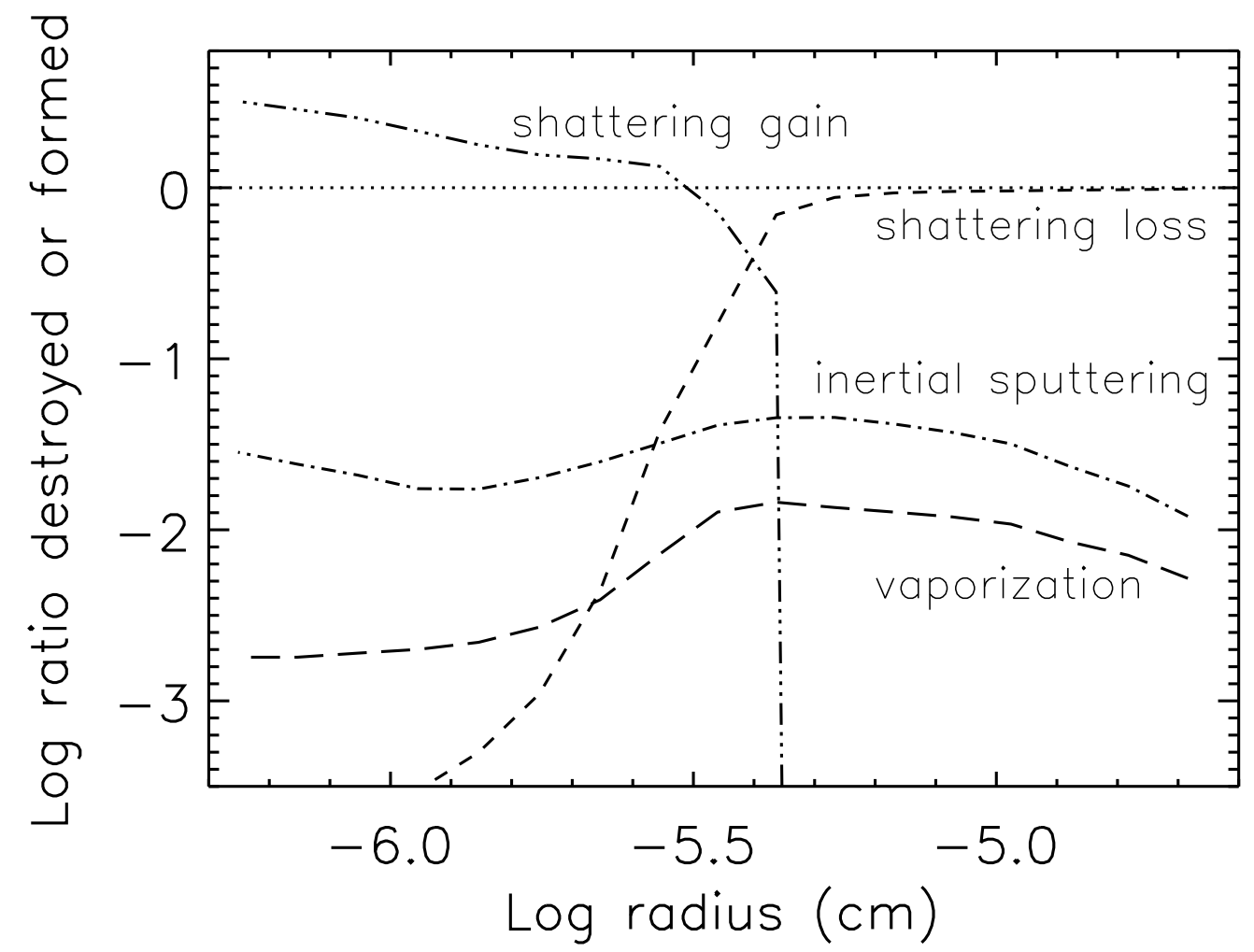

Figure 3: Postshock graphite grain destruction, disruption and formation as a function of radius, given as the ratio of initial to final mass at a given radius, for vaporization (long-dashed), inertial sputtering (dashed-dotted), shattering mass loss (short-dashed), and shattering mass gain (dashed-triple dotted) in a $100 \mathrm{~km} \mathrm{~s}^{-1}$ shock.

supernova shocks predominates. This study uses up-to-date algorithms for the dust processing in shocks [34], and for the first time includes the effects of shattering in grain-grain collisions in shock waves [35]. In the calculations we assumed a preshock density of $n_{\mathrm{H}}=0.25 \mathrm{~cm}^{-3}$, a temperature of $T_{\mathrm{k}}=8000 \mathrm{~K}$, a component of the interstellar magnetic field normal to the shock front of $B_{0}=3 \mu \mathrm{G}$, and an initial MRN size distributions of graphite and silicate grains. We modeled the effects of thermal and inertial sputtering of grains, and vaporization and shattering in graingrain collisions in shocks of velocities $v_{\mathrm{s}}=50,100,150$, and $200 \mathrm{~km} \mathrm{~s}^{-1}$, and a range of preshock densities. In Figure 3 we present the processing of graphite grains as a function of grain radius for a $100 \mathrm{~km} \mathrm{~s}^{-1}$ shock, and in Figure 4 we show the preshock and postshock grain size distributions for shocks of velocity 50,100 , and $200 \mathrm{~km} \mathrm{~s}^{-1}$. These figures clearly show the dominant effects of grain shattering in redistributing the grain mass from large grains $(a>500 \AA)$ into smaller fragments, and also the effects of inertial sputtering in grain destruction. Vaporization makes a relatively minor contribution to grain destruction.

In Figure 5 we show the probability for interstellar grains to survive the passage of a supernova shock wave. This has been calculated as $a_{\text {half }}$, the grain radius for 


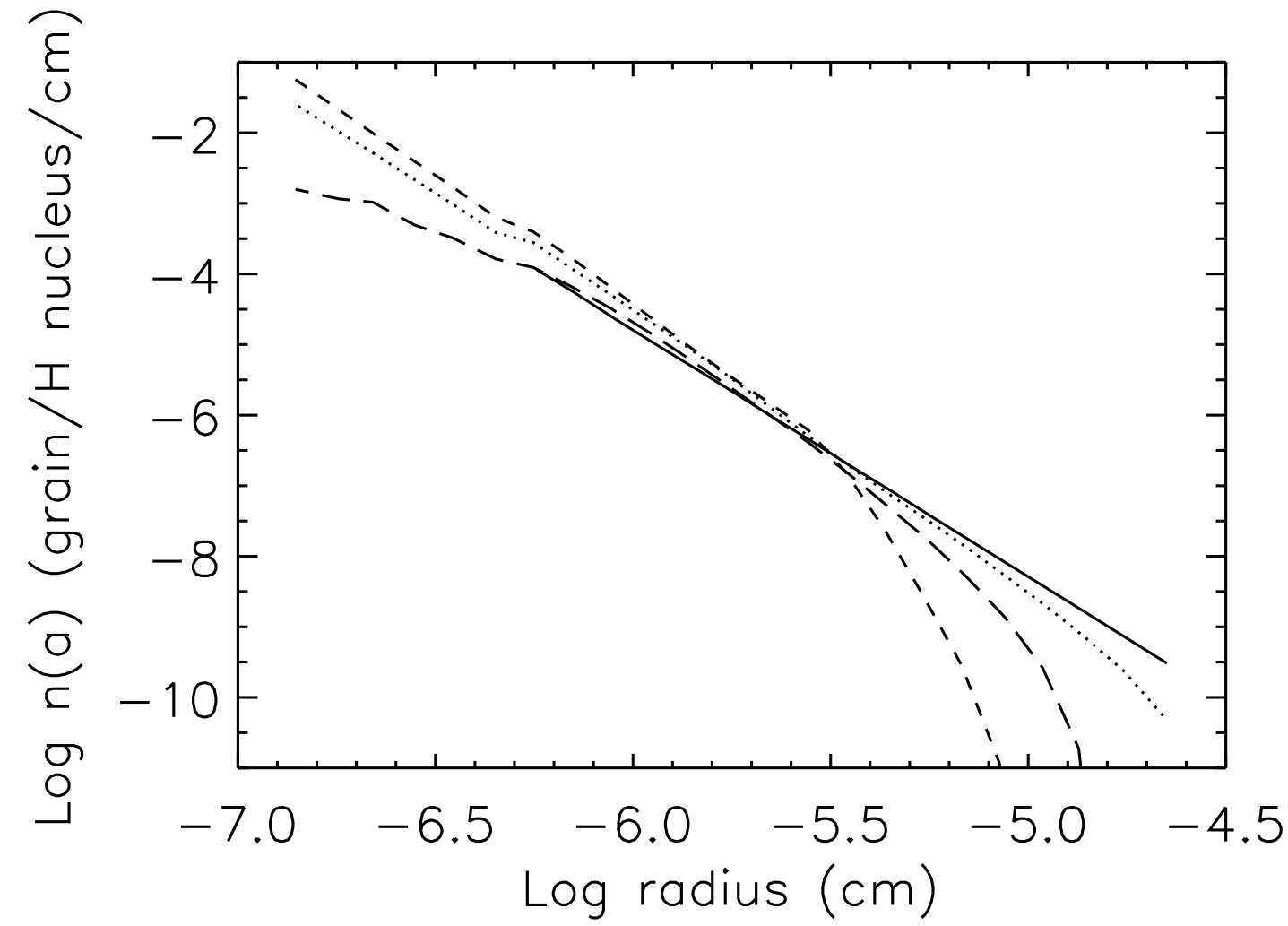

Figure 4: Graphite grain initial MRN size distribution (solid), and postshock size distributions for shock velocities of $50 \mathrm{~km} \mathrm{~s}^{-1}$ (dotted), $100 \mathrm{~km} \mathrm{~s}^{-1}$ (short-dashed), and $200 \mathrm{~km} \mathrm{~s}^{-1}$ (long-dashed). The preshock size distribution ranges from $50 \AA$ to $2500 \AA$; -6.3 to -4.6 in Log radius $(\mathrm{cm})$.

which half of the initial grains survive. For grains with radii larger than $a_{\text {half }}$ less than half of the original grains survive the passage of the shock, and for grains smaller than $a_{\text {half }}$ more than half of the grains survive. From this one can see that large grains $(a>1000 \AA)$ have a relatively high probability of surviving the passage of a $50 \mathrm{~km} \mathrm{~s}^{-1}$ shock, but this probability decreases rapidly for higher shock velocities.

We can calculate the time scale $t_{\text {SNR }}$ for supernova shock waves to destroy interstellar dust (i. e., to return the grain mass to the gas as atoms) in all phases of the ISM [21]:

$$
t_{\mathrm{SNR}}=\frac{9.7 \times 10^{7}}{\int \epsilon\left(v_{\mathrm{s} 7}\right) / v_{\mathrm{s} 7}^{3} d v_{\mathrm{s} 7}} \quad \mathrm{yr},
$$

where $v_{\mathrm{s} 7}$ is the shock velocity (in units of $100 \mathrm{~km} \mathrm{~s}^{-1}$ ), and $\epsilon\left(v_{\mathrm{s} 7}\right)$ is the efficiency of grain destruction for a shock of velocity $v_{\mathrm{s} 7}$.

From calculated grain destruction data one can derive analytical expressions for $\epsilon\left(v_{\mathrm{S} 7}\right)$ for the graphite and silicate MRN grain populations [22,35]. The time scales to return the entire grain mass to the gas as atoms are $t_{\mathrm{SNR}}=6.3 \times 10^{8} \mathrm{yr}$ and $3.7 \times 10^{8}$ yr for graphite and silicate MRN size distributions, respectively. The destruction of small grains $(<500 \AA)$ is dominated by high velocity $\left(>250 \mathrm{~km} \mathrm{~s}^{-1}\right)$ 


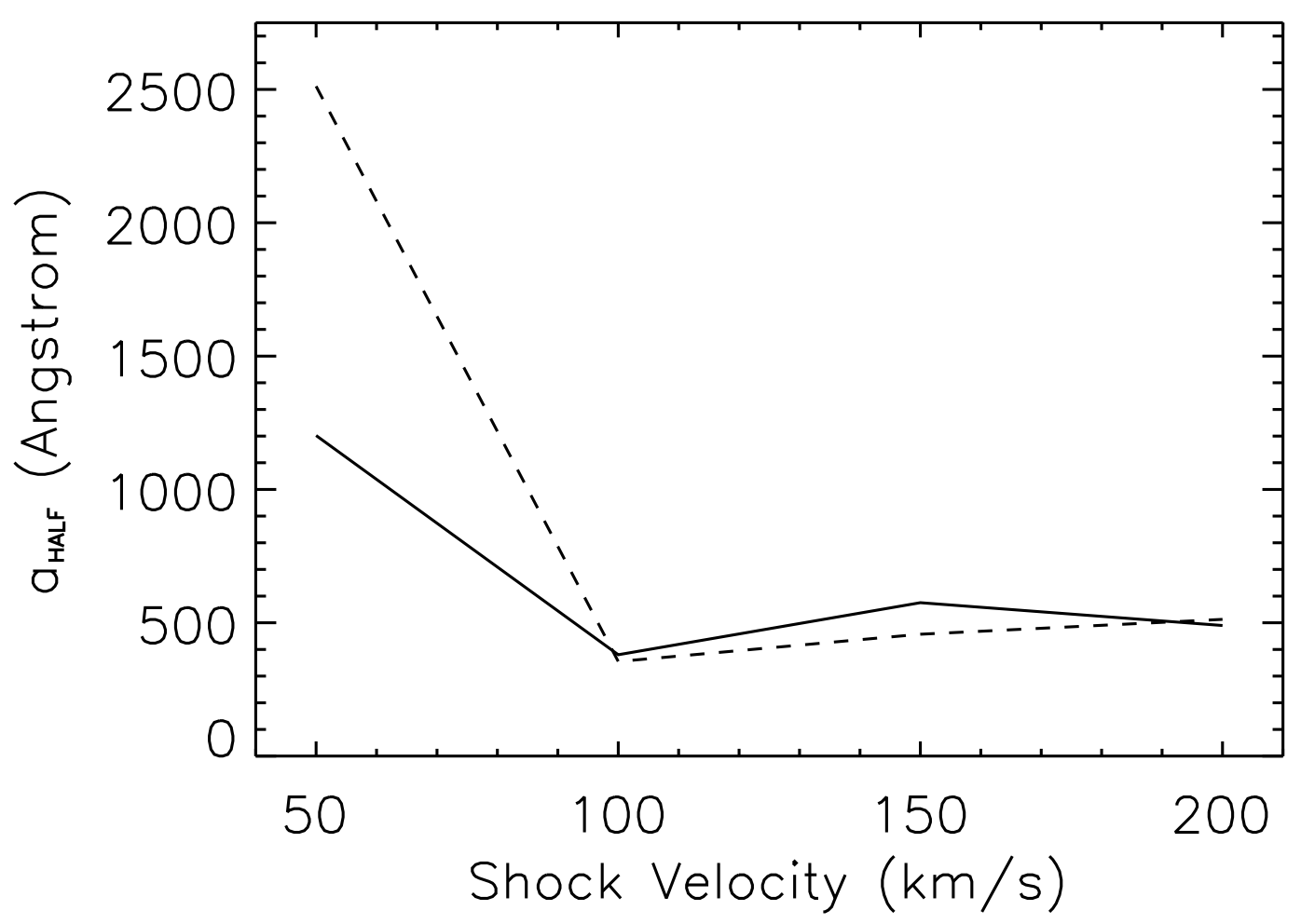

Figure 5: Grain radius for which $50 \%$ of the initial grains survive the passage of a single shock, $a_{\mathrm{HALF}}$, plotted against the shock velocity, for graphite/amorphous carbon grains (solid line) and silicate grains (dashed line). Grains larger than $a_{\mathrm{HALF}}$ have a probability of less than half of surviving the passage of a single shock.

shocks. Thermal sputtering in such a shock removes a layer of equal thickness, $\Delta a$, from all grains. For a $300 \mathrm{~km} \mathrm{~s}^{-1}$ shock $\Delta a$ is of order $300 \AA$ [20]. Thus, while high velocity shocks are very infrequent (once every $\simeq 6 \times 10^{8}$ yr), they essentially completely destroy all grains with sizes less than $\Delta a$. For large grains, betatron acceleration is important and shocks at all velocities contribute about equally to the total destruction rate. In this case, the small fraction destroyed by a single low velocity shock, as compared to a high velocity shock, is compensated for by the much higher frequency of low velocity shocks. The average time between shocks of velocity $v_{\mathrm{s} 7}$ is given by $10^{8} v_{\mathrm{s} 7}^{2}$ years [20]. In our earlier studies that did not include the effects of shattering large grain destruction was dominated by vaporization in grain-grain collisions in low velocity shocks $\left(v_{\mathrm{s}}=50 \mathrm{~km} \mathrm{~s}^{-1}\right)$, and by sputtering in higher velocity shocks. In the latest models large grains are shattered in grain-grain collisions in the surface layers of shocks $\left(50 \mathrm{~km} \mathrm{~s}^{-1} \leq v_{\mathrm{s}} \leq 200 \mathrm{~km} \mathrm{~s}^{-1}\right)$. In high velocity shocks $\left(v_{\mathrm{s}} \geq 200 \mathrm{~km} \mathrm{~s}^{-1}\right)$ the increased surface area to mass ratio, due to the fragmentation of the large grains, leads to much greater destruction by thermal sputtering in the hot postshock gas [35], compared to the earlier models without shattering [22].

The inclusion of shattering in the shock code increases the derived grain life- 
Table 3: Interstellar Dust Lifetimes, $t_{\mathrm{SNR}}$, and Disruption Time Scales, $t_{\mathrm{dd}}$

\begin{tabular}{lcc} 
& \multicolumn{2}{c}{$t_{\text {SNR }}\left(\times 10^{8}\right.$ year $)$} \\
\hline Component & Carbon & Silicate \\
\hline MRN & 6.3 & 3.7 \\
\hline$\geq 1000 \AA$ & $\leq 0.5$ & \multicolumn{1}{c}{$t_{\mathrm{dd}}\left(\times 10^{8}\right.$ year $)$} \\
\hline MRN $=$ & Mathis, Rumpl \& Norsieck $(1977)$ \\
& $\begin{array}{l}\text { size distribution, } d n(a) \propto a^{-3.5} d a, \\
\text { with } 50 \AA \leq a \leq 2500 \AA\end{array}$ \\
$\geq 1000 \AA \begin{array}{l}\text { all grains in the MRN size } \\
\text { distribution with radii } \geq 1000 \AA\end{array}$
\end{tabular}

times slightly, compared to calculations without shattering, because the large grains are shattered into smaller particles that survive better in shocks. However, these lifetimes still fall short of the dust lifetimes of $t_{\mathrm{SNR}} \geq 2 \times 10^{10} \mathrm{yr}$ [22] required to preserve $\geq 90 \%$ of the stellar ejected silicates in the ISM $[5,44]$. Therefore, the well-known conundrum remains $[18,22,37,39-43]$ : Why is silicate dust, which may contain as much as $90 \%$ of the available silicon, so abundant in the ISM? Moreover, a marginally increased grain lifetime is now accompanied by the almost complete disruption of large interstellar grains $(1000 \AA \leq a \leq 2500 \AA)$. Shattering in grain-grain collisions therefore adds a new conundrum: Why do visual extinction measurements show that most of the mass in interstellar dust is in large grains (radii $\geq 1000 \AA$ )?

Shattering in grain-grain collisions leads to the almost complete elimination of large grains $(a \geq 1000 \AA)$ from the MRN dust population in a fast shock $(v \sim$ $\left.100 \mathrm{~km} \mathrm{~s}^{-1}\right)$. The disruption time scales, $t_{\mathrm{dd}}$ - the time scale to disrupt all large

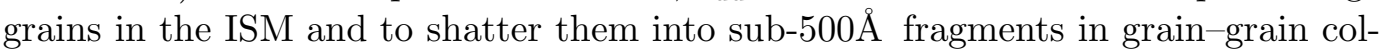
lisions in shock waves - are considerably shorter than the destruction time scales. The derived grain lifetimes, $t_{\mathrm{SNR}}$, and large-grain disruption time scales, $t_{\mathrm{dd}}$, are summarized in Table 3. The disruption time scales are factors 12 (graphite) and 6 (silicate) times smaller than the destruction lifetimes. We conclude from this that large graphite and large silicate grains have short disruption time scales in the diffuse ISM. This implies that large interstellar grains must reform in the ISM. This can occur by the condensation of volatile gas phase species onto grain surfaces (accretion), or by the coagulation of the grains into large porous structures. Accretion alone cannot cannot reform large grains because of the limited supply of condensible gas phase species. The accretion of icy mantles may, however, aid coagulation by increasing the probability of particle coalescence in grain-grain collisions [45]. The monomers or cores making up these composite aggregates are likely have radii less than about $500 \AA$, as indicated by Figure 5 . 


\section{Mitigating Effects on Dust Processing in the ISM}

All theoretical studies of dust processing in the ISM contain certain assumptions about the values of physical parameters that are not well-determined observationally or experimentally. It is therefore worthwhile to consider and discuss some of these uncertainties, and to see how they may effect the derived time scales for dust processing in the ISM. These regions of uncertainty fall into three broad categories, namely: the structure of the ISM, the structure of supernova remnant shocks, and the microphysical properties of the dust. In this section we discuss some of the uncertainties in these parameters and their effects on the dust processing time scales.

Firstly, with regard to the structure of the ISM, the volume filling factors for the different components, and the cycling time scales between the components are not well-determined observationally. For example, in our calculations $[1,22,35]$ we have assumed an idealized multiphase model of the ISM [17], and the results of these calculations depend on the parameters of the ISM adopted in the model. Most of the dust destruction occurs in the warm intercloud gas, but the amount of dust destruction is not very sensitive to the model of the ISM, being about the same for both two-phase and three-phase models [21]. The rate of dust destruction would be reduced if there were substantially less dust in the warm gas than in the cold gas, but this is not the case [46]. Although the gaseous abundances of refractory elements are significantly higher in the warm gas than in the cold gas, most of the refractory material remains tied up in dust in both phases. The calculations of the dust processing time scale linearly with the adopted supernova rate. Some supernovae occur in stellar associations; since later supernovae occur within the bubbles created by earlier supernovae in these associations, such supernova will be less effective in destroying dust. This effect was allowed for in our estimate of the dust destruction time, which is based on [21]: we have used an effective supernova rate in the Galaxy of 1 per 125 years, substantially less than the actual rate of about 1 per $40 \mathrm{yr}$ (for $\mathrm{H}_{0}=75 \mathrm{~km} \mathrm{~s}^{-1} \mathrm{Mpc}^{-1}$ [47]).

Secondly, dust destruction is directly affected by the structure of supernova remnant shock waves. For shock velocities greater than about $200 \mathrm{~km} \mathrm{~s}^{-1}$ the shock is non-radiative and the amount of dust destroyed depends upon the long-term evolution of the remnant. Thermal conduction and cloud evaporation both reduce the temperature of the shocked gas and thereby reduce dust destruction by thermal sputtering, the dominant dust destruction process in such shocks. Slower shocks are radiative, and the amount of dust destroyed depends upon the strength of the magnetic field [37]. If the Galactic magnetic field is about $5 \mu \mathrm{G}$ instead of $3 \mu \mathrm{G}$ [48], then the dust destruction in radiative shocks will be reduced by a factor of about 2 [22]; the overall reduction is then substantially less than the factor of 8 that has been suggested [49]. The calculations undertaken to date assume that the supernova remnant expands into a uniform medium or a uniformly cloudy medium. Massive stars will create low density cavities surrounded by shells of gas [50], and it is not known yet how this will affect the amount of grain destruction. 
Thirdly, the microphysical properties of the dust will directly determine its processing time scales. Recent theoretical studies of the sputtering of astrophysically relevant materials suggest much lower yields near threshold [51] than our semiempirical sputtering formalism predicts [34]. For carbonaceous grains, much of the discrepancy reflects a difference in the adopted binding energy $(7.4 \mathrm{eV}$ appropriate for graphite [51] versus $4 \mathrm{eV}$ appropriate for amorphous carbon [52]). It is well established experimentally that graphite will amorphitize under the effects of ionbombardment and, thus, it has been argued [34] that the lower binding energy is the correct one to use, even if the preshock dust is assumed to be graphite. For silicates, the origin of the discrepancy is not well understood but it should be noted that the theoretical method may be suspect near threshold [53]. In the end, these differences in the adopted binding energies or sputtering yields near threshold do not influence the derived dust life time very much. While the exact threshold influences the minor fraction of dust returned to the gas at low shock velocities, the dust lifetime measures the contribution from all shocks where the grain velocities may substantially exceed this threshold, and in this case the influence of the threshold is minimal. In fact, while silicate and carbon grains have binding energies of $5.7 \mathrm{eV}$ and $4 \mathrm{eV}$ in our calculations, the calculated lifetime of silicate grains is slightly shorter than for carbon $\left(4 \times 10^{8}\right.$ yr versus $\left.6 \times 10^{8} \mathrm{yr}\right)$, reflecting the difference in specific density and hence stopping length behind the shock [22]. In principle, the structure of interstellar grains (i. e., porosity) has more consequences for the calculated lifetimes. The mass to cross-section ratio affects the grain stopping length, and hence its destruction, because the drag on the grains varies as $(a \rho)^{-1}$. Moreover, porous aggregate particles may be disrupted (shattered) early-on in the shock and, therefore, are not betatron accelerated. This will limit the destruction of such grains in radiative shocks $\left(v_{\mathrm{s}}<200 \mathrm{~km} \mathrm{~s}^{-1}\right)$. However, by the same token, the much larger surface area offered by the disrupted aggregates will lead to greater thermal sputtering in non-radiative shocks $\left(v_{\mathrm{s}}>200 \mathrm{~km} \mathrm{~s}^{-1}\right)$. While we have yet to carry out a full calculation of these effects, we anticipate that the difference in the derived dust lifetimes will be less than a factor two.

Clearly, there are many variables in the determination of dust processing time scales in the ISM that need to be much better determined, e.g., the structure and evolution of supernova remnants and the physical processing of porous dust particles in shock waves. Therefore, it is in these directions that future studies of the ISM and dust processing within it could most usefully be directed. Nevertheless, as emphasized in this review, dust lifetimes in the ISM are predicted to be $\simeq 5 \times 10^{8}$ yr, much shorter than the stardust injection time scale of $\simeq 3 \times 10^{9}$ yr [1]. As a direct consequence, dust reformation in the ISM is a necessary condition for the evolution of interstellar dust. 


\section{Discussion}

Figure 3 shows that graphite (or amorphous carbon) grains, lose $2-5 \%$ of their mass, or a fraction of their radius $\Delta a / a \simeq 0.7-1.7 \%$, due to inertial sputtering in a $100 \mathrm{~km} \mathrm{~s}^{-1}$ shock. This primarily occurs through the process of sputtering by gas atoms and ions during slowing down with respect to the gas. The mass loss due to inertial sputtering is relatively insensitive to the grain size. Essentially, grains collide with approximately their own mass of gas during the slowing down process. Betatron acceleration increases this mass loss somewhat for the larger grains for shock velocities, $v>50 \mathrm{~km} \mathrm{~s}^{-1}$. For SiC grains the inertial sputtering yield is about $50 \%$ higher than for graphite [34], and so $\Delta a / a$ will be of order $1.0-2.2 \%$. Diamond materials have similar sputtering yields to graphite, and so the degree of inertial sputtering for the interstellar diamonds will be the same as for graphite, i. e., $\Delta a / a \simeq$ $0.7-1.7 \%$.

For large grains shattering due to grain-grain collisions has a profound influence. The largest grains considered in shock calculations are generally those defined by a MRN size distribution, i.e., of order a few thousand $\AA$. These grains are rapidly disrupted into smaller grain fragments (see $\S 4$ ), and so are predicted to be rapidly removed from the interstellar size distribution on time scales of order a few tens of millions of years by shocks in the warm ISM [35]. They are primarily disrupted by catastrophic grain-grain collisions; collisions in which more than half of the larger (target) grain mass is shattered into sub- $300 \AA$ fragments. However, interstellar grains with sizes $\gg 1 \mu \mathrm{m}$ will fare somewhat better in interstellar shocks because of their large mass and the low abundance of collision partners large enough to cause their catastrophic fragmentation in grain-grain collisions. In general, the main agent for the disruption of a moving target grain is a collision with a grain which is just large enough for the kinetic energy of the impact to catastrophically shatter the whole grain. For a $5 \mu \mathrm{m} \mathrm{SiC} \mathrm{grain} \mathrm{at} 100 \mathrm{~km} \mathrm{~s}^{-1}$, this grain limiting size is $\simeq 0.8 \mu \mathrm{m}$ as compared to a limiting size of $500 \AA$ for a typical interstellar grain of $2500 \AA$. Because the interstellar grain size distribution steepens considerably above $a=3000 \AA$, a $5 \mu \mathrm{m}$ grain will have a very low probability for such a catastrophic collision to occur. Such large grains will still being cratered by impacts with the smallest grains and lose $\simeq 5 \%$ of their mass. Thus, large grains travel relatively unscathed through interstellar shocks. In contrast, because of their lower intrinsic strength, large $(<10 \mu \mathrm{m})$ graphite or silicate grains will be disrupted in shocks.

It is an inescapable conclusion of the analysis presented here that presolar grains should retain some memory of their sojourn through the ISM, i. e., traces of exposure to sputtering in the gas phase and surface pitting due to the sub-catastrophic (or cratering) impact of small grains in shock waves. The inferred ages for the recovered $\mathrm{SiC}$ grains are of the order of hundreds of millions of years [54] much larger than the typical interval between supernovae shock waves $\left(\sim\right.$ few $\times 10^{7}$ years $)$. The extracted presolar grains would therefore be expected to show some traces of their passage through the ISM. However, it appears that the extracted grain surfaces seem to be relatively clean, and that many of the particles have crystallographic faces (Berna- 
towicz these proceedings), which is clearly in contrast to the expectations from the discussion above. It should, nevertheless, be pointed out that in extracting the presolar grains from the meteoritic material they undergo very extreme oxidation processes, which may in part modify the original surfaces of the particles. Perhaps the surfaces seen in the laboratory are not the same faces that were exposed to the ISM? It is also possible that in the general ISM the grains are mantled with some other material. This could perhaps explain why the SiC absorption feature at $11.3 \mu \mathrm{m}$ has only been seen in circumstellar environments; outside these regions the presence of absorbing mantles of carbon could suppress the feature [55]. The most likely solution, particularly for the large graphite grains, is that the recovered grains are those that have never been exposed to interstellar shock waves; i.e., the grains that survive are the (few) grains which never saw a strong shock in the first place. This is also true for the small grains (i. e., the diamonds) which are relatively unaffected by low velocity shocks $\left(<250 \mathrm{~km} \mathrm{~s}^{-1}\right)$ but are completely destroyed by the first high velocity shock they encounter. With a fast shock time scale of $3 \times 10^{8}$ yr and a star-ISM cycle time of $3 \times 10^{9} \mathrm{yr}$, an average sample of the ISM would contain $\simeq 10 \%$ of material which never saw such a fast shock.

\section{Conclusions}

Stellar winds are the initial injectors of circumstellar dust from its site of formation into the ambient ISM. The action of the stellar radiation on the dust, and its collisions with the gas are relatively benign resulting in very little processing during this stage in its evolution. However, stellar winds are probably not able to propagate the dust over large interstellar distances. For dust distribution over large interstellar scales we require that the effects of supernovae play a major role. Supernova shock waves can drive large masses of gas and dust through the ISM, but they also heavily process or even destroy the bulk of the entrapped dust.

Within the context of the sizes and composition of the extracted meteoritic presolar grains we can now draw some interesting conclusions. These grains must have undergone some processing in supernova shock waves in the warm ISM, the phase in which dominant grain destruction occurs. The results of model calculations show


can survive passage through shock waves $\left(v_{\mathrm{s}}<200 \mathrm{~km} \mathrm{~s}^{-1}\right)$ relatively unharmed. They merely lose of order $1-2 \%$ of their outer layers, which for a $100 \AA$ radius grain would be less than one atomic layer, and therefore of minimal consequence. On the other hand thermal sputtering in fast shocks does destroy small grains efficiently. However, $\simeq 10 \%$ of the interstellar grains are incorporated into stars and planetary systems before they encounter such a fast shock. For micron-sized SiC grains the dominant surface processing will be due to inertial sputtering, because of their great resistance to cratering in collisions with small grains. However, for micron-sized graphite grains the effects of cratering should dominate. Perhaps these differences are reflected in the nature of the meteoritic presolar grain surfaces? However, the 
indications from extensive sample analyses are that the surfaces of the recovered presolar grains are unprocessed. Does this result really imply that the grains have not been processed in the ISM, or that the surfaces of the analyzed grains are not the faces that were exposed in the ISM? Perhaps modification in the laboratory, during the rigorous extraction processes, has removed the original surfaces? What is now seen could be some underlying layer that was protected by overlying layers, or by mantles of material deposited in the ISM. In order to address these questions concerning the surface processing of dust in the ISM it would be useful to perform some control experiments on laboratory-made micron-sized particles. It is possible that during the extraction of interstellar grains from meteorites some surface alteration may occur. The same oxidative extraction processes used to recover interstellar meteoritic grains should be applied to well-characterized $\mathrm{SiC}$ and graphite particles, in order to quantify the degree of surface alteration that occurs during the extraction process.

The presolar grains extracted from meteorites indicate that these grains can survive for long time scales in the ISM, survive the process of incorporation into the solar nebula, and also survive eventual capture into meteoritic bodies in the solar system. However, it must be remembered that the extracted presolar grains consist of highly refractory materials, and are thus a very selective sample of the dust in the general ISM. Also, the extracted dust may represent a small fraction of the interstellar stardust which did survive shock processing. The bulk of the interstellar grain materials, and those for which we have observational evidence, e.g., amorphous silicates, and by inference also amorphous carbon, have not yet been extracted from the meteorites and analyzed. This is probably no easy task, given their less resilient nature. Capturing interstellar grains in space and returning them to Earth for analysis is required if we are to obtain a more representative sample.

Acknowledgements. APJ wishes to thank the McDonnell Center for the Space Sciences and NASA for the invitation to attend this conference. Theoretical studies of interstellar dust at NASA Ames are supported under RTOP 399-20-10-13 from the astrophysics theory program. The research of CFM is supported in part by NSF grant AST95-30480. 


\section{References}

1. Jones, A.P., \& Tielens, A.G.G.M. 1994, in The Cold Universe, XIIIth Moriond Astrophysics Meeting, ed. T. Montmerle, C.J. Lada, I.F. Mirabel, \& J. Tran Thanh Van (Gif-sur Yvette: Editions Frontieres), 35

2. Jones, A.P. 1997, in From Stardust to Planetesimals, ed. Y.J. Pendleton, \& A.G.G.M. Tielens, Astronomical Society of the Pacific, Conference Series, in press

3. Cherchneff, I., \& Tielens, A.G.G.M. 1994, in Circumstellar Media in the Late Stages of Stellar Evolution, ed. R.E.S. Clegg and W.P.S. Meikle, (Cambridge University Press), p232

4. Moore, M.H., Tanabe, T., \& Nuth, J.A. 1991, ApJ, 373, L31

5. Mathis, J.S. 1990, ARA\&A, 28, 37

6. Mathis, J.S., Rumpl, W., \& Nordsieck, K.H. 1977, ApJ, 217, 105 (MRN)

7. Tielens, A.G.G.M. 1990, in Carbon in the Galaxy, ed. J.C. Tarter, S. Chang, \& D. DeFrees (NASA Conf. Publ. 3061), 59

8. Kim, S.-H., Martin, P.G., \& Hendry, P.D. 1994, ApJ, 422, 164

9. Whittet, D.C.B. 1992, Dust in the Galactic Environment, Institute of Physics Publishing, Bristol.

10. Millar, T.J., \& Williams, D.A. (eds.) 1993, Dust and Chemistry in Astrophysics, Institute of Physics Publishing, Bristol

11. Evans, A. 1994, The Dusty Universe, John Wiley, Chichester

12. Cutri, R.M., \& Latter, W.B. (eds.) 1994, The First Symposium on the Infrared Cirrus and Diffuse Interstellar Clouds, San Francisco, A.S.P. Conference Series 58

13. Nenner, I. (ed.) 1994, Molecules and Grains in Space, AIP Conf. Proc. 312, AIP Press, New York

14. Greenberg, J.M. (ed.) 1995, The Cosmic Dust Connection, Kluwer, Dordrecht

15. Dorschner, J., \& Henning, T. 1995, A\&A Reviews, 6, 271

16. Pendleton, Y.J., \& Tielens, A.G.G.M. (eds.) 1997, From Stardust to Planetesimals Astronomical Society of the Pacific, Conference Series

17. McKee, C.F., \& Ostriker, J.P. 1977, ApJ, 218, 148

18. Draine, B.T., \& Salpeter, E.E. 1979, ApJ, 231, 438

19. Dwek, E., \& Scalo, J.M. 1979, ApJ, 233, L81

20. Seab, C.G. 1987, in Interstellar Processes. ed. D.J. Hollenbach \& H.A. Thronson Jr., (Dordrecht: Reidel), 491

21. McKee, C.F. 1989, in Interstellar Dust. ed. L.J. Allamandola \& A.G.G.M. Tielens, (Dordrecht, Kluwer), 431

22. Jones, A.P., Tielens, A.G.G.M., Hollenbach, D.J., \& McKee, C. F. 1994, ApJ, 433, 797

23. Bradley, J.P. 1994, Science, 265, 925

24. Martin, P.G. 1995, ApJ(Letters), 445, L63

25. Anders, E., \& Zinner, E. 1993, Meteoritics, 28, 490

26. Draine, B.T. 1995, in The Physics of the Interstellar Medium and Intergalactic Medium, A. Ferrara, C.F. McKee, C. Heiles, \& P.R. Shapiro (eds.), Astronomical Society of the Pacific, Conference Series, Vol. 80, 133 
27. Grün, E., Zook, H.A., Baguhl, M., Balogh, A., Bame, S.J., Fechtig, H., Forsyth, R., Hanner, M.S., Horanyi, M., Kissel, J., Lindblad, B.-A., Linkert, D., Linkert, G., Mann, I., McDonnell, J.A.M., Morfill, G.E., Phillips, J.L., Polanskey, C., Schwehm, G., Siddique, N., Staubach, P., Svestka, J., \& Taylor, A. 1993, Nature, 362, 428

28. Grün, E., Gustafson, B., Mann, I., Baguhl, M., Morfill, G.E., Staubach, P., Taylor, A., \& Zook, H.A. 1994, A\&A, 286, 915

29. Taylor, A., Baggaley, W.J., \& Steel, D.I. 1996, Nature, 380, 323

30. Tielens, A.G.G.M. 1983, ApJ, 271, 702

31. Dominik, C., Gail, H.-P., \& Sedlmayr, E. 1989, A\&A, 223, 227

32. Habing, H.J., Tignon, J., \& Tielens, A.G.G.M. 1994, A\&A, 286, 523

33. Jura, M. 1987, in Interstellar Processes, ed. D.J. Hollenbach \& H.A. Thronson, (Dordrecht; Reidel), 3

34. Tielens, A.G.G.M., McKee, C.F., Seab, C.G., \& Hollenbach, D.J. 1994, ApJ, 431, 321

35. Jones, A.P., Tielens, A.G.G.M., \& Hollenbach, D.J. 1996, ApJ, 469, 740

36. Woitke, P., Dominik, C., \& Sedlmayr, E. 1993, A\&A, 274, 451

37. McKee, C.F., Hollenbach, D.J., Seab, C.G., \& Tielens, A.G.G.M. 1987, ApJ, 318,674

38. Borkowski, K.J., \& Dwek, E. 1995, ApJ, 454, 254

39. Barlow, M.J. 1978, MNRAS, 183, 367

40. Barlow, M.J. 1978, MNRAS, 183, 397

41. Draine, B.T., \& Salpeter, E.E. 1979, ApJ, 231, 77

42. Dwek, E., \& Scalo, J.M. 1980, ApJ, 239, 193

43. Seab, C.G., \& Shull, J.M. 1983, ApJ, 275, 652

44. Draine, B.T., \& Lee, H.K. 1984, ApJ, 285, 89

45. Chokshi, A., Tielens, A.G.G.M., \& Hollenbach, D. 1993, ApJ, 407, 806

46. Savage, B.D., \& Sembach, K.R. 1996, ApJ, 470, 893

47. van den Bergh, S., \& McClure, R.D. 1994, ApJ 425, 205

48. Boulares, A., \& Cox, D.P. 1990, ApJ, 365, 544

49. Crinklaw, G., Federman, S.R., \& Joseph, C.L. 1994, ApJ, 424, 748

50. McKee, C.F., Van Buren, D., \& Lazareff, B. 1984, ApJ 278, L115

51. Flower, D.R., Pineau des Forêts, G., Field, D., \& May, P.W. 1996, MNRAS, 280,447

52. Benson, S.W. 1976, Thermochemical Kinetics (New York: Wiley)

53. Tielens, A.G.G.M. 1997, in Formation and Evolution of Solids in Space, ed. J.M. Greenberg, in press

54. Lewis, R.S., Amari, S., \& Anders, E. 1990, Nature, 348, 293

55. Kozasa, T., Dorschner, J., Henning, T., \& Stognienko, R. 1996, A\&A, 307, 551 